\title{
Novel Innovation Model of E-Commerce Consumption Based on Big Data Processing and Analysis Technology
}

\author{
Hongsheng $\mathrm{Xu}^{1,2} \mathrm{a}^{*}$, Ganglong Fan ${ }^{1,2}$ and Yanqing $\mathrm{Lv}^{1,2}$ \\ ${ }^{1}$ Luoyang Normal University, Luoyang, 471934, China \\ ${ }^{2}$ Henan key Laboratory for Big Data Processing \& Analytics of Electronic Commerce, Luoyang, \\ 471934, China \\ a85660190@qq.com
}

\begin{abstract}
Keywords: Big data; Data analysis; E-commerce consumption model; Data acquisition; Data processing
\end{abstract}

\begin{abstract}
Big data is a large volume, data category is particularly large data sets, and such data sets can not use traditional database tools to capture, manage and deal with its content. Big data value chain can be divided into 4 stages: data generation, data acquisition, and data storage and data analysis. Data analysis aims at extracting useful values, providing judgments, suggestions, or supporting decisions. By analyzing data sets from different fields, different levels of potential values can be generated. The paper presents novel innovation model of e-commerce consumption based on big data processing and analysis technology.
\end{abstract}

\section{Introduction}

For enterprise organizations, the value of big data is reflected in two aspects: analysis, use and development of the two. Analyzing large data can reveal hidden information. For example, retail sales of stores, geographic and social information can enhance understanding of customers. Two times the development of big data is that successful Internet companies. For example, Facebook customized a highly personalized user experience by combining a large amount of user information and created a new advertising model. This kind of commercial behavior that creates new products and services through big data is not a coincidence.

With the advent of the cloud era, big data has attracted more and more attention. Big data is often used to describe a large amount of unstructured and semi-structured data created by a company that spends too much time and money on downloading relational databases for analysis. Large data analysis is often associated with cloud computing because real-time large data set analysis requires a framework like MapReduce to distribute tasks to dozens, hundreds, or even thousands of computers.

One of the advantages of comparing e-commerce and traditional retailing based on Internet is the availability of data. E-commerce allows real-time access to the source of the customer's visit, the search, collection, buying behavior, and the purchase of goods within the site. These data can help enterprises more accurately for customer service [1]. The development of artificial intelligence, information systems and decision science has promoted a variety of analytical methods and tools, including data mining, customer behavior models, decision support, and so on.

Big data, also known as big data refers to the amount of data involved is huge to not through the current mainstream software tools to capture, management, processing and finishing to become more active to help business decision-making purposes of information within a reasonable time. The earliest reference to the term "big data" dates back to Apache org's open source project [2]. At that time, large data was used to describe a large collection of data needed for simultaneous batch processing or analysis to update the network search index.

Big data because the body is constantly increasing, the value density units of data on the decline, but the overall value of the data is on the rise. Some big data is equivalent to gold and oil, said the big data contains the infinite commercial value. 
In the retail industry, technology and method of data analysis is widely used, traditional enterprises such as WAL-MART through data mining to reshape and optimize the supply chain, the rise of new electricity providers such as Amazon through the understanding and analysis of massive data, provide more professional and personalized service for users. In such an era, if companies and manufacturers ignore their views, they will lose a lot of attention to the crowd, and the influence of the traditional marketing model will be greatly reduced. The paper presents novel innovation model of e-commerce consumption based on big data processing and analysis technology.

\section{Big Data Key Technologies of Data Processing in the Cloud Era}

Big data has changed the pattern of traditional management decision structures. Studying the impact of big data on the management decision structure will be an open research issue. In addition, changes in decision structures require people are to explore how to do "two" mining for supporting higher levels of decision-making". No matter what kind of data heterogeneity is brought about by big data, the "rough knowledge" in big data can still be regarded as the category of "one mining". It is necessary to search for the "intelligent knowledge" produced by "two mining" as a bridge between data heterogeneity and decision heterogeneity. Exploring how the decision structure is changed in a large data environment is equivalent to studying how the subjective knowledge of the decision maker is involved in the decision-making process.

Internet search engine is one of the most typical applications of big data. Baidu daily processing data reached dozens of $\mathrm{PB}$, and showed a high growth trend. If one disk has a capacity of $1 \mathrm{~GB}$, it amounts to tens of millions of CDs built together. Microsoft Bing (in China, Bing) search engine, a week to respond to 10 billion orders of magnitude search request. Sensing equipment, more mobile terminal access network, the data generated and its growth rate is much more than any time in history, the data traffic on the Internet is growing rapidly [3].

With the coming of the cloud era, the main body of data creation is gradually shifted from enterprise to individual, and the vast majority of data produced by individuals are unstructured data such as pictures, documents and videos. The popularization of information technology enables more office flows to be realized through the network, and the data generated are mainly unstructured data.

Now, when the amount of data accumulation is large enough, the quantitative change has caused qualitative change. "Big data" through massive data for analysis, given the Internet "IQ", which makes the role of the Internet, from simple data transfer and information exchange, up to the massive data analysis based on the phrase "he started thinking". In short, the big data is to break the mass of data in a certain period of time to complete the screening, analysis, and organize into useful information to help users complete the decision-making. With big data, decision makers can quickly perceive market demand changes, thus enabling them to make more favorable decisions for the enterprise, which makes them more innovative and competitive. This is following the cloud computing and networking IT industry a disruptive technological change, the national governance model of corporate decision-making, organization and business processes, personal life will have a huge impact, as is shown by equation (1) [4].

$$
F_{P}=a_{1 m} X_{1}+a_{2 m} X_{2}+\cdots+a_{p m} X_{P}
$$

The most common is the information stored in the form of text, such as e-mail communication, company files to the web pages, social media content etc.. Therefore, text analysis is considered to have more commercial potential than structured data mining. Typically, text analysis, also known as text mining, refers to the process of extracting useful information and knowledge from unstructured text. Text mining is an interdisciplinary field that involves information retrieval, machine learning, statistics, and computational linguistics, especially in data mining.

Big data, in terms of personal privacy, a large amount of data often contains some detailed, potentially revealing information about us, which gradually raises our concerns about personal privacy. Some big data companies need to take this issue seriously. For example, the United States Teradata left 
people with a deep impression is one of his scientists, we should not obey the privacy legal protection simply, these are not enough, the company should comply with the principle of Google is not evil, even more should make more positive efforts.

SOA's three data center models are data as a service (DaaS) model, physical hierarchy model and architecture component model. The DaaS data access model describes how the data is provided to the SOA component. The physical model describes how the data is stored and how the stored hierarchical map is transmitted to the SOA data store. Finally, the architecture model describes the relationships between data, data management services, and SOA components.

Information consumption has become the most active consumption hot spot in our country in recent years. The principle of the development of information consumption industry by the State Council is "market orientation, reform push, demand guidance and orderly safety [5]. The state is making great efforts to promote the development of information consumption. According to the Ministry of statistics, the first half of last year, the national information consumption continues to expand the scale; the scale reached 2 trillion yuan, an increase of $20.7 \%$.

Total e-commerce transactions amounted to 49800, an increase of 45.3\%. Infrastructure investment capacity continued to increase, the cumulative completion of fixed investment 129 billion 650 million. New information services and applications continue to emerge, as is shown by equation (2), where it is the application of intelligent products has become increasingly prominent, and e-commerce transactions continue to grow at a high speed. Not only the scale of the information consumption market itself is expanding, but the information consumption also directly related to the rapid growth of related industries.

$$
\left\{\begin{array}{l}
\frac{d I}{d S}=-1+\frac{1}{\sigma S} \\
I_{0}+S_{0}+R_{0}=1
\end{array}\right.
$$

Data acquisition: ETL tools will be responsible for the distributed heterogeneous data sources, the data such as relational data, and data files from plane to temporary middle layer after cleaning, transformation and integration, and finally loaded into the data warehouse and data mart, online analysis become the foundation treatment, data mining [6]. Data access: relational database, NOSQL, SQL and so on. Infrastructure: cloud storage, distributed file storage, etc.. Data processing: Natural Language Processing (NLP) is a discipline that deals with the language of human computer interaction.

In the process of collecting data, the main characteristics and challenges is the high number of concurrent, because at the same time there may be tens of thousands of users to access and operate, such as train ticketing website and Taobao, visit their concurrent at the peak reached millions, so in the end need to support the deployment of a large number of data acquisition. And how to load and distribute between these databases really needs deep thinking and design.

\section{Novel Innovation Model of E-Commerce Consumption Based on Big Data Processing and Analysis Technology}

Big data is the rapid data collection and analysis, to obtain valuable information and technical ability, mainly including data acquisition, storage, management, analysis and visualization of mining technology and its integration in diverse or huge amounts of data [7]. To tap the value of big data necessary for analysis and calculation is on the contents of big data. Deep learning and knowledge is the basis for calculation of large data analysis, and analysis of the key technology of data visualization is also the results of data analysis show the key technology.

Although the acquisition terminal itself will have a lot of database, but if the effective analysis of these data, and these should be from the front end of the data into a centralized large-scale distributed database, or distributed storage cluster, and can do some simple cleaning and pretreatment in the import basis. Some users also use the Storm from the Twitter to stream data on the part of the import to meet the real-time computing requirements of some businesses. The characteristics and challenges of the 
introduction and the pretreatment process is mainly imported a large amount of data, the import amount per second will often reach hundreds of megabytes, or even Gigabit level.

Big data processing technology in the specific application, and can provide technical support and processing platform for national pillar enterprises for enterprise data analysis, data analysis, processing, mining, extracting important information and knowledge, and then converted into useful model, applied to the research, production, operation and sales process [8]. At the same time, the State advocates a "smart city" construction in the city, and information fusion background, focus on improving people's livelihood, enhance the competitiveness of enterprises, promote the sustainable development of the city and other concerns, the comprehensive utilization of networking, cloud computing and other information technologies, based on existing information fusion City, city operation service concept advanced city.

The establishment of information network coverage and depth of interconnection, resources, environment, infrastructure, industry and other factors on the city's comprehensive perception, and integration [9]. City information platform to build collaborative sharing, for intelligent processing of information utilization, thus providing intelligent response control for city operation and the allocation of resources, to provide intelligent decision basis and means for the government's social management and public service, as is shown by equation(3), where it is the integrated development of the regional informatization process of intelligent information resources and open information platform for enterprises and individuals.

$$
x_{i+1}^{2}(t+1)=\left(1-d_{i}^{2}(t)\right) x_{i}^{2}(t)+\left(\frac{N^{2}(t)}{N^{3}(t)+N^{2}(t)}\right) s_{i} \alpha N^{1}(t)
$$

As a new form of innovation, business model innovation is no less important than technological innovation. In recent years, business model innovation has become a popular word in Chinese business circles. Business model innovation is refers to the enterprise value creation and innovation changes provide the basic logic, which may include changes in multiple elements of business model; the change of it may also include the relationship between the elements or dynamic mechanism. Generally speaking, business model innovation means that enterprises make money in a new and effective way.

This is the first step in changing the business model of big data, such as capturing, storing and analyzing the after-sales experience that users publish on social media, to improve quality and improve services. The enterprise should not only capture and store large data, but also should develop and use big data, because only the development and utilization of big data, to dig out the value of big data is huge, especially should use special tools to analyze and develop a cluttered, unstructured data. Understanding of consumer sentiment, optimize the supply chain, the removal of false data, therefore, enterprises should invest in infrastructure and software, using the corresponding algorithm to handle large data, and employ data scientists to complete the corresponding work [10]. Only by compressing data and intelligently displaying data related to specific content, can we make better use of big data.

SOA and all kinds of data model in the level of integrated data model, data collection hidden in a set of abstract data server, the server has one or more interfaces connected to the application, also provides all the integrity and function of data management. Components do not directly access data, but as a form of service, just as they do in simple cases, the data requirements are purely RDBMS models. Application components are basically divorced from the differences in data management between RDBMS and large data. Although for the above reasons, this method cannot create a simple RDBMS query model, but it replicates at least the simple RDBMS model we mentioned above.

The electronic commerce enterprise scale increasing, enterprises need to its core business data analysis, can no longer feel intuition or make critical decisions, the best of all customer related business data analysis, in order to retain existing customers and attract them to buy more goods, at the same time to win more new customers. It adopts the distributed technology and combines a series of technologies, which can analyze the massive data in real time, and meet the needs of a part of the large data environment. But I say this is wrong, at least one-sided, is not able to completely solve the big data storage management needs. Cloud computing will have a huge impact on the development of relational 
databases, while most large business systems and e-commerce systems use databases based on relational databases.

\section{Summary}

The paper presents novel innovation model of e-commerce consumption based on big data processing and analysis technology. Business model innovation is more systematic and fundamental, and it is not a single factor change. It often involves many factors in the business model, and at the same time great changes require a larger strategic adjustment of the enterprise organization. It is a kind of integrated innovation.

\section{Acknowledgements}

This paper is supported by Henan key Laboratory for Big Data Processing \& Analytics of Electronic Commerce, and also supported by the science and technology research major project of Henan province Education Department (13B520155, 17B520026).

\section{References}

[1] Meng XF, Ci X. Big Data Management: Concept, Techniques and Challenges. Journal of Computer Research and Development, 2013, 50(1): 146-169.

[2] Barham P, Dragovic B, Fraser K, Hand S, Harris T, Ho A, Neugebauer R, Pratt I, Warfield A. Xen and the art of virtualization. ACM SIGOPS Operating Systems Review, 2015, 37(8): 164-177.

[3] Wei Li, Hongtu Zhang, Tingting An, "Optimal Decision-Making in E-Commerce Platform Based On Optimal Stopping Theory", JCIT, Vol. 8, No. 8, pp. $922 \sim 929,2013$.

[4] Zaharia M, Chowdhury M, Das T, Dave A, Ma J, McCauley M, Franklin MJ, Shenker S, Stoica I. Resilient distributed datasets: A fault-tolerant abstraction for in-memory cluster computing, Proceedings of the 9th USENIX conference on Networked Systems Design and Implementation. USENIX Association, 2012: 2-2.

[5] ZHANG Rui-ling, XU Hong-sheng, "Building and mapping ontology of e-business based on fuzzy rough concept lattices”, Journal of Convergence Information Technology, vol.6, no.9, (2011), pp. 81-88.

[6] Chang F, Dean J, Ghemawat S, Hsieh WC, Wallach DA, Burrows M, Chandra T, Fikes A, Gruber RE. Bigtable: A distributed storage system for structured data. In: Proc. of the 7th USENIX Symp. on Operating Systems Design and Implementation. Berkeley: USENIX Association, 2006. 205-218.

[7] Rodrigo Rocha Silva, Celso Massaki Hirata, Joubert de Castro Lima, Computing BIG data cubes with hybrid memory, JCIT, Vol. 11, No. 1, pp. 13 -30, 2016.

[8] Dean J, Ghemawat S. MapReduce: simplified data processing on large clusters. Communications of the ACM, 2014, 26(6): 107-113.

[9] Zaharia M, Chowdhury M, Franklin M J, Shenker S, Stoica I. Spark: cluster computing with working sets,Proceedings of the 2nd USENIX conference on Hot topics in cloud computing. 2013: $10-10$.

[10] Kang Xiang-ping,Li De-yu,Wang Su-ge, "Rough set model based on formal concept analysis", Information Sciences, vol. 222, (2013), pp.611-625. 